\title{
Rhabdomyolysis and Neuroleptic Malignant Syndrome Associated with Very Low-dose Antipsychotics in Children and Adolescent
}

\author{
Jong-Il Park', Tae Won Park ${ }^{1,2}$ \\ ${ }^{1}$ Department of Psychiatry, Chonbuk National University Medical School, ${ }^{2}$ Research Institute of Clinical Medicine of Chonbuk National \\ University-Biomedical Research Institute of Chonbuk National University Hospital, Jeonju, Korea
}

\begin{abstract}
Along with the field of adult psychiatry, antipsychotic agents are increasingly used in the field of child and adolescent psychiatry. Although neuroleptic malignant syndrome (NMS) and rhabdomyolysis are rare complication associated with antipsychotic agent, clinicians should need to pay attention to all potential adverse drug reaction (ADR). Also, ADRs in child and adolescent could show different signs and symptoms compared with those in adult. In this case report, we present a case of NMS in a child which occurred shortly after the resolution of rhabdomyolysis which was induced by low-dose risperidone.
\end{abstract}

KEY WORDS: Neuroleptic malignant syndrome; Rhabdomyolysis; Risperidone.

\section{INTRODUCTION}

Along with the field of adult psychiatry, antipsychotic agents are increasingly used in the field of child and adolescent psychiatry with various psychiatric disease. ${ }^{1)}$ However, many of the clinical use with antipsychotic agents in child and adolescent is empirical and not based on sufficient randomized controlled trials. Children is highly susceptible to adverse drug reactions (ADRs) induced by antipsychotics; such as weight gain, extrapyramidal symptoms, hyperprolactinaemia, and even serious adverse reaction including rhabdomyolysis and neuroleptic malignant syndrome (NMS). ${ }^{2,3)}$ Dealing with ADRs of antipsychotics is significant issues since ADRs of antipsychotics are associated with low subjective wellbeing of treated patients. ${ }^{4)}$ The first step in dealing with ADRs would be early detection. However, there are some different clinical manifestrations in child and adolescent which make it difficult to detect rhabdomyolysis and

Received: April 11, 2018/Revised: July 5, 2018

Accepted: July 23, 2018

Address for correspondence: Tae Won Park

Department of Psychiatry, Chonbuk National University Medical

School, 20 Geonji-ro, Deokjin-gu, Jeonju 54907, Korea

E-mail: ptaewon@jbnu.ac.kr

ORCID: https://orcid.org/0000-0001-5980-0756
NMS. In this case report, we could highlights the clinical manifestation of rhabdomyolysis and NMS in child and adolescent. To our best knowledge, this report is the first case of NMS in a child which occurred shortly after the resolution of rhabdomyolysis which is induced by lowdose risperidone.

\section{CASE}

A 13-year-old Asian male diagnosed with post traumatic stress disorder (PTSD) began to undergo psychiatric treatment using sertraline $25 \mathrm{mg}$ and risperidone $0.25 \mathrm{mg}$ due to anxiety, reliving traumatic experiences, hypervigilance, episodes of intermittent explosions, and impulsivity after experiencing violence and ostracization in his school. Wechsler Intelligent Scale for Children (WISC) showed that the patient's full-scale intelligence quotient was 56 . The patient was obese with a body mass index of $36.7 \mathrm{~kg} / \mathrm{m}^{2}$. The patient was not taking medications other than drugs for psychiatric symptoms.

On the fifth day of risperidone administration, the patient showed irritability, paranoid ideation, and sleep disturbance in addition to existing PTSD symptoms; he was promptly admitted for further evaluation and treatment. We increased the dose of risperidone to $0.5 \mathrm{mg}$, and we

(ㄷ) This is an Open-Access article distributed under the terms of the Creative Commons Attribution Non-Commercial License (http://creativecommons.org/licenses/by-nc/4.0) which permits unrestricted non-commercial use, distribution, and reproduction in any medium, provided the original work is properly cited. 
also added quetiapine $25 \mathrm{mg}$ and lorazepam $1 \mathrm{mg}$ to the treatment regimen. On the second day of admission, routine laboratory examination showed a mild liver function test elevation (aspartate aminotransferase/alanine transaminase, 41/48 IU/L) and creatine kinase (CK, 1,181 IU/L) without elevation of creatinine or myoblobin. The physical examination did not reveal any symptoms other than abdominal pain, and there was no history related to CK elevation, such as excessive physical activity, seclusion, alcohol drinking, or trauma. Electrolyte was in normal ranage ( $\mathrm{Na}, \mathrm{K}, \mathrm{Cl}: 139 \mathrm{mmol} / \mathrm{L}, 3.8 \mathrm{mmol} / \mathrm{L}, 100 \mathrm{mmol} / \mathrm{L})$. The patient was referred to the department of internal medicine and diagnosed with mild rhadbomyolysis without significant renal failure (creatinine, $0.81 \mathrm{mg} / \mathrm{dl}$; estimated glomerular filtration rate, $132.5 \mathrm{ml} / \mathrm{min} / 1.73 \mathrm{~m}^{2}$; all within the normal range). He was treated conservatively with hydration. We administered sertraline 25 $\mathrm{mg}$, risperidone $0.75 \mathrm{mg}$, lorazepam $1 \mathrm{mg}$, and quetiapine $12.5 \mathrm{mg}$ to the patient to treat psychiatric symptoms for the duration of admission. The laboratory findings were normalized and the patient was discharged on the 22nd day of admission.

At first outpatient follow-up, the patient exhibited a firm face with hostile attitude and was admitted again. On the first day, the patient was administered sertraline $50 \mathrm{mg}$, risperidone $0.75 \mathrm{mg}$, lorazepam $1 \mathrm{mg}$, and quetiapine $12.5 \mathrm{mg}$. The patient complained of abdominal pain, similar to when he was first admitted. At that night, the patient exhibited disorientation, inappropriate behaviors, irritability, and sleep disturbance. Since the patient was not controlled, intramuscular haloperidol $2.5 \mathrm{mg}$ and lorazepam $2 \mathrm{mg}$ was administered at the dawn of second day. Laboratory findings showed CK elevation to $739 \mathrm{IU} / \mathrm{L}$ again. In addition, the patient began to exhibit fever $\left(38.1^{\circ} \mathrm{C}\right)$ and tachycardia (heart rate, 137 beats per minute). The dose of antipsychotics were increased to risperidone $1 \mathrm{mg}$ and quetiapine $25 \mathrm{mg}$ for control of irritability and sleep disturbance. The patient exhibited sustained fever $\left(39.1^{\circ} \mathrm{C}\right)$, blood pressure fluctuation, and even altered consciousness. On the third day of admission, we stopped antipsychotic medications and the patient was transferred to the pediatric intensive care unit due to the worsening symptoms. On the fourth day of admission, the CK level was elevated to 4,587 IU/L. We performed brain magnetic resonance imaging and lumbar puncture to rule out central nervous system infection and inflammation; however, the result was negative. There was no lesion that may have caused secondary hypertension in terms of patient's blood pressure elevation other than fatty liver shown from the abdomen computed tomography. Conservative treatments, including hydration and dantrolene and bromocriptine administrations, were performed for NMS. The vital signs of the patient began to stabilize, and other laboratory examination results, including the $\mathrm{CK}$ level, gradually became normal. The patient exhibited sleep disturbance and irritability during his stay in the pediatric ward, and he was administered quetiapine $12.5 \mathrm{mg}$, low-potency antipsychoatics, and lorazepam $0.5 \mathrm{mg}$ on the 19th day of admission. After discharge, the patient has been stable on quetiapine and lorazepam for six months.

\section{DISCUSSION}

Rhabdomyolysis and NMS related to antipsychotic medications are rare, but such conditions could cause death if not treated properly. NMS is a fatal complication of antipsychotic drugs and shows up to $18.8 \%$ mortality. In this case report, we present a case of NMS in a child which occurred shortly after the resolution of rhabdomyolysis which associated with antipsychotics. To the best of our knowledge, the dose of antipsychotics (risperidone $0.25 \mathrm{mg}$ ) used in our case was the lowest dose associated with rhabdomyolysis and NMS. Regarding our patient, rhabdomyolysis was discovered on the sixth day of administering risperidone and the conservative treatment for rhabdomyolysis began. However, the cause of rhabdomyolysis was not adequately understood since the symptoms for rhabdomyolysis were mild. The patient was discharged as CK level was normalized, while he was on risperidone and quetiapine. A few days after discharge with continuous use of antipsychotics, CK elevation was reappeared and finally NMS symptoms were observed.

This observation suggests that risperidone is the causal factor of rhabdomyolysis and later NMS. The causality of our case was assessed using the Naranjo ADR probability scale, ${ }^{6}$ ) which scored 8 (probable ADR). Although it is unclear, the use of intramuscular haloperidol for uncontrolled agitation, which was observed during the night of second admission, might have worsened NMS caused by the use of risperidone. Among the three antipsychotics used in this case, quetiapine treatment had been continued for six months after discharge without any compli- 
cations including NMS or rhabdomyolysis. Thus, quetiapine was less likely considered as the causal factor for ADRs.

The incidence of NMS yielded an overall estimate of 0.991 cases per thousand people $(140 / 141,291){ }^{7)}$ The incidence of rhabdomyolysis or/and NMS associated with the use of antipsychotics in child adolescent population might be lower than those of adults, but only case reports and a limited number of case series were available in this population. The lower incidence might be associated with the situation that ADR in child and adolescent population are reported lower than those of adults or that the majority of the prescription antipsychotics are used to treat adult patients. As the prescription of antipsychotics to treat several psychiatric disorders in pediatric patients is increasing, ${ }^{8)}$ a deeper understanding of the ADRs related to the use of antipsychotics in pediatric patients should be needed. Pediatric patients may be more sensitive to ADR than adults. Moreover, because symptoms of pediatric patients manifest differently from adults, a closer observation is warranted. Thus, the reasons that hindered suspicion and the early discovery of rhabdomyolysis and NMS should be considered. The first clinical symptoms of rhabdomyolysis which we did not recognize was abdominal pain in our patient. We might consider that rhabdomyolysis in pediatric patients may manifest as abdominal pain or cramps in the early stage ${ }^{2,9)}$ and that antipsychotic drugs are one of the causal agent in rhabdomyolysis. Second, although fever and CK elevation existed earlier, a lack of rigidity delayed NMS detection. As shown in this case, many NMS cases in pediatric patients are not manifested as full-blown as in adult patients. The research of NMS associated with antipsychotics ${ }^{10)}$ reported that only $39 \%$ (9 of 23) of cases exhibit rigidity, whereas all cases exhibited CK elevation. Further structured, systematic studies on the effects and ADRs that occur with increasing use of antipsychotics are required for enhanced understanding.

\section{Conflicts of Interest}

No potential conflict of interest relevant to this article was reported.

\section{REFERENCES}

1. Kim BN. The clinical use of risperidone in child \& adolescent psychiatry. Clin Psychopharmacol Neurosci 2003;1(Suppl): 178-187.

2. Star K, lessa N, Almandil NB, Wilton L, Curran S, Edwards IR, et al. Rhabdomyolysis reported for children and adolescents treated with antipsychotic medicines: a case series analysis. J Child Adolesc Psychopharmacol 2012;22:440-451.

3. Rani FA, Byrne PJ, Murray ML, Carter P, Wong IC. Paediatric atypical antipsychotic monitoring safety (PAMS) study: pilot study in children and adolescents in secondary- and tertiary-care settings. Drug Saf 2009;32:325-333.

4. Lee YW, Kim JH, Ann JH, Cho SE, Lee J, Kim MJ, et al. Subjective well-being in patients with schizophrenia treated with atypical antipsychotics: the impact of psychopathology and adverse drug effects. Clin Psychopharmacol Neurosci 2010:8:149-155.

5. Shalev A, Hermesh $\mathrm{H}$, Munitz $\mathrm{H}$. Mortality from neuroleptic malignant syndrome. J Clin Psychiatry 1989;50:18-25.

6. Naranjo CA, Busto U, Sellers EM, Sandor P, Ruiz I, Roberts EA, et al. A method for estimating the probability of adverse drug reactions. Clin Pharmacol Ther 1981;30:239-245.

7. Gurrera RJ, Simpson JC, Tsuang MT. Meta-analytic evidence of systematic bias in estimates of neuroleptic malignant syndrome incidence. Compr Psychiatry 2007;48:205-211.

8. Zito JM, Safer DJ, DosReis S, Gardner JF, Magder L, Soeken K, et al. Psychotropic practice patterns for youth: a 10-year perspective. Arch Pediatr Adolesc Med 2003; 157:17-25.

9. Mannix R, Tan ML, Wright R, Baskin M. Acute pediatric rhabdomyolysis: causes and rates of renal failure. Pediatrics 2006; 118:2119-2125.

10. Neuhut R, Lindenmayer JP, Silva R. Neuroleptic malignant syndrome in children and adolescents on atypical antipsychotic medication: a review. I Child Adolesc Psychopharmacol 2009; 19:415-422. 